\title{
ORIGINAL ARTICLE Forty-five-year follow-up on the renal function after spinal cord injury
}

This article has been corrected since advance online publication and a corrigendum is also printed in this issue.

\author{
M Elmelund ${ }^{1,2,3}$, PS Oturai ${ }^{4}$, B Toson ${ }^{5}$ and F Biering-Sørensen ${ }^{1,2}$
}

Study design: Retrospective chart review.

Objectives: To investigate the extent of renal deterioration in patients with spinal cord injury (SCl) and to identify risk indicators associated with renal deterioration.

Setting: Clinic for Spinal Cord Injuries, Rigshospitalet, Hornbæk, Denmark.

Methods: This study included 116 patients admitted to our clinic with a traumatic SCl sustained between 1956 and 1975. Results from renography and ${ }^{51} \mathrm{Cr}$-EDTA plasma clearance were collected from medical records from time of injury until 2012, and the occurrence of renal deterioration was analysed by cumulative incidence curves. The impact of demographics, neurological level and completeness of $\mathrm{SCl}$, urinary tract stones, dilatation of the upper urinary tract (UUT) and bladder-emptying methods were analysed with Cox proportional hazard ratios.

Results: The bladder-emptying methods used for the longest period were reflex triggering (63\%), bladder expression (22\%), indwelling catheter (5\%), normal voiding (4\%), ileal conduit (3\%) and clean intermittent catheterisation (2\%). The cumulative risk of moderate renal deterioration (functional distribution outside $40-60 \%$ on renography or relative glomerular filtration rate (GFR) $\leqslant 75 \%$ of expected according to age and gender) was $58 \%$. The cumulative risk of severe renal deterioration (functional distribution outside $30-70 \%$ on renography or relative GFR $\leqslant 51 \%$ ) was $29 \%$ after 45 years postinjury. Only dilatation of UUT and renal/ureter stone requiring removal significantly increased the risk of moderate and severe renal deterioration.

Conclusion: Renal deterioration occurs at any time after injury, suggesting that lifelong follow-up examinations of the renal function are important, especially in patients with dilatation of UUT and/or renal/ureter stones.

Spinal Cord (2016) 54, 445-451; doi:10.1038/sc.2015.242; published online 12 January 2016

\section{INTRODUCTION}

Patients with spinal cord injury (SCI) have an increased risk of renal deterioration and urinary tract complications, and historically, renal deterioration was the primary cause of death in SCI patients. Since the 1970s, renal-related mortality in SCI patients has decreased markedly, partly owing to improved management of the bladder-emptying method, but recent studies show that renal deterioration remains a predictor of all-cause mortality in SCI patients. ${ }^{1,2}$ Today, physicians agree that patients with SCI should have their renal function evaluated, but there is no consensus on time intervals for long-term follow-up examinations, and different recommendations on renal examinations have been proposed. ${ }^{3,4}$ Most SCI care centres offer follow-up examinations of the upper urinary tract (UUT) and renal function every year or second year, including renography, radiolabelled tracer clearance, creatinine clearance, serum creatinine, ultrasound, computed tomographic (CT) scan or intravenous pyelography. ${ }^{5}$ To avoid unnecessary examinations, reduce exposure to radiation and limit health-care expenses, it could be questioned whether the period between examinations should be longer or whether routine examinations should terminate after a certain period of time with a stable level of the renal function.
In order to determine the optimal follow-up program of the renal function in SCI patients, knowledge of the prevalence and time to onset of renal deterioration after SCI is essential. Most studies describing the renal function and urological complications focus on the first years after SCI. To our knowledge, the longest published follow-up after injury is 29.3 years. ${ }^{6}$ In addition, most studies evaluate urinary tract complications rather than the renal function.

The aim of this study was to describe the extent of renal deterioration in patients with SCI over a 45-year follow-up period. A secondary aim was to identify potential risk indicators associated with renal deterioration in SCI patients.

\section{MATERIALS AND METHODS}

\section{Study population}

We reviewed the medical records of all patients, admitted to the Clinic of SCI, Rigshospitalet, Hornbæk, Denmark, with a traumatic SCI sustained during the period 1944-1975. In total, 154 patients were registered. To be included in the study, patients should have attended the follow-up examinations of the renal function offered in the clinic for $\geqslant 7$ consecutive years and should as a minimum have had one renography carried out. Of the complete cohort of 154 patients, there were no records on 13 patients, 7 patients did not have a SCI and 18 patients did not meet the inclusion criteria. Eleven of the 18 excluded

${ }^{1}$ Clinic for Spinal Cord Injuries, Rigshospitalet, Hornbæk, Denmark; ${ }^{2}$ Faculty of Health and Medical Sciences, University of Copenhagen, Copenhagen, Denmark; ${ }^{3}$ Department of Obstetrics and Gynecology, Herlev University Hospital, Herlev, Denmark; ${ }^{4}$ Department of Clinical Physiology, Nuclear Medicine and PET, Rigshospitalet, Copenhagen, Denmark and ${ }^{5}$ Neuroscience Research Australia (NeuRA), Sydney, New South Wales, Australia

Correspondence: Dr M Elmelund, Clinic for Spinal Cord Injuries, Rigshospitalet, Havnevej 25, Hornbæk 3100, Denmark.

E-mail: marleneelmelund@hotmail.com

Received 7 September 2015; revised 14 December 2015; accepted 15 December 2015; published online 12 January 2016 
patients had moved to another part of the country or abroad after the SCI. A total of 116 patients with a traumatic SCI in the period 1956-1975 were included in the study, as shown in Figure 1. Further details regarding the study population are described in the study by Elmelund et al. ${ }^{7}$

\section{Procedures}

Most patients attended follow-up examinations of the renal function every second year lifelong. From medical records, we obtained results from all urological and renal examinations, as well as the bladder-emptying methods used. Until the 1980s, follow-up examinations included plasma creatinine measurements and X-ray of the abdomen or intravenous pyelography. From the 1980s, CT scan and renography were carried out routinely. The majority of renographies were performed with ${ }^{99 \mathrm{~m}} \mathrm{Tc}$-labelled mercaptoacetyltriglycine three, although dimercaptosuccinic acid, diethylentriamine pentaacetic acid (DTPA) and ${ }^{131}$ I-hippuran scans were also included in the study. Glomerular filtration rate (GFR) measurement, cystoscopy, additional UUT imaging, voiding cystourethrography and urodynamic investigations were performed when considered necessary, usually when the renography or plasma creatinine measurement was abnormal. GFR measurements were performed with plasma clearance determination of a radiolabelled tracer, in most cases with a single injection of ${ }^{51} \mathrm{Cr}$-EDTA. In this study, GFR results were converted to an age- and gender-adjusted relative GFR ( $r$-GFR). ${ }^{8,9}$

The primary outcome of this study was renal deterioration, based on results from renography and GFR measurements. In accordance with the previously published article, two different definitions of renal deterioration were used: ${ }^{7}$ (1) moderate renal deterioration, with a split renal function on renography $\leqslant 40 \%$ in one kidney or a r-GFR measurement $\leqslant 75 \%$ of expected according to age and gender; and (2) severe renal deterioration, with a split renal function on renography $\leqslant 30 \%$ in one kidney or $\mathrm{r}-\mathrm{GFR} \leqslant 51 \%$ of expected according to age and gender.

Several variables were investigated: calendar year of injury, age at injury, gender, neurological level of injury, completeness of injury categorised by the Frankel grade, ${ }^{10}$ predominant bladder-emptying method, dilatation of UUT, and occurrence of bladder stones or renal/ureter stones, with or without removal. We investigated whether there was an association between the variables and moderate or severe renal deterioration as well as the most abnormal r-GFR for the 83 patients with a GFR measurement.

As most patients changed their bladder-emptying method throughout the 45-year follow-up period, each patient was assigned a predominant method, as introduced by Weld and Dmochowski ${ }^{11}$ and $\mathrm{Ku}$ et al..$^{6}$ : the predominant bladder-emptying method used for the longest period since injury. For those patients experiencing renal deterioration, the predominant bladder-emptying method used for the longest period until first abnormal renal examination was included in the analyses.

The presence or absence of dilatation of UUT was determined by intravenous pyelography, CT scan, ultrasound examination or by delayed excretion on renography, as implied by time to max $\geqslant 6 \mathrm{~min}$ and/or $>50 \%$ residual renal activity after $20 \mathrm{~min}$ on renographic time-activity curves, excluding renography with DTPA. The occurrence of bladder, ureter or renal stones was investigated

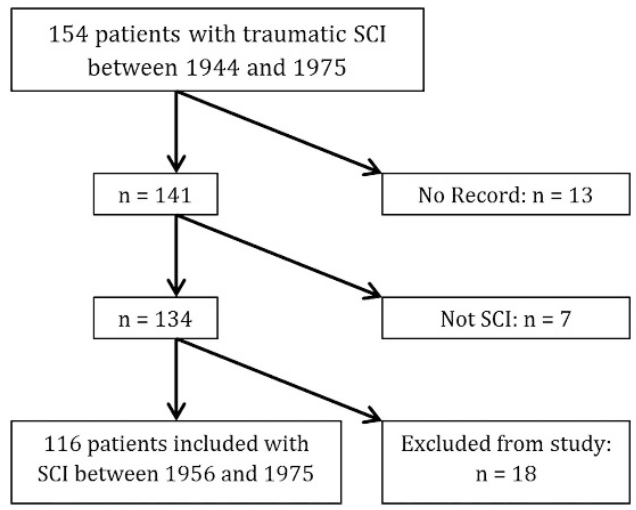

Figure 1 Selection of $\mathrm{SCl}$ patients in the study. with X-ray, intravenous pyelography, ultrasound, CT scan or cystoscopy. The removal of ureter/renal stones included endoscopic removal and open surgery, pyelolitomy and extracorporeal shockwave lithotripsy.

\section{Statistical analysis}

All statistical analyses were performed using SAS (version 9.3; SAS Institute, Cary, NC, USA) and Microsoft Excel 2011 (Microsoft, Redmond, WA, USA) software packages. Cumulative incidence analyses were used to examine the risk of renal deterioration over time, and Cox proportional hazards models were used to investigate the effect of different covariates on the renal function. The assumptions for using proportional hazards models were analysed for each covariate by the use of Wald test with a time-dependent covariate and the $S$ choenfeld residual plots. Unpaired $t$-tests and analysis of variance were used to compare r-GFR values for each covariate, and a linear regression analysis was conducted to investigate the multivariate effect of selected variables on $\mathrm{r}$-GFR. Only $P$-values $<0.05$ were considered significant.

\section{RESULTS}

\section{Study population}

Patient demographics, including causes of death as defined by Frankel et al., ${ }^{1}$ are shown in Table 1 .

Several patients had urological surgical procedures performed during the follow-up period. Four patients had an external sphincterotomy and six patients, who used reflex voiding, had transurethral resection of the internal sphincter/bladder neck incision carried out. There were six patients with only one functional kidney: two patients had a nephrectomy owing to traumatic kidney damage in relation to

Table 1 Characteristics of patients with spinal cord injuries, mean ( \pm s.d.) or numbers (\%)

No. of patients 116

\section{Gender \\ Female \\ Male}

$16(13.8 \%)$ $100(86.2 \%)$

Age at injury (years) $22( \pm 8)$

Follow-up (years after injury)

$39( \pm 7)$

Level of neurological lesion

Cervical

$54(46.6 \%)$

Thoracic

$48(41.4 \%)$

Lumbar

$14(12.0 \%)$

\section{Completeness (Frankel grade)}

A

$49(42.2 \%)$

B

$28(24.1 \%)$

C

D

$13(11.2 \%)$

$24(20.7 \%)$

$2(1.7 \%)$

Death during follow-up

$42(36.2 \%)$

Cause of death ${ }^{\mathrm{a}}$

$\begin{array}{ll}\text { Pneumonia/other respiratory causes } & 10(23.8 \%)\end{array}$

Cancer

Septicaemia

Heart disease

Injuries

$3(7.1 \%)$

Urinary system diseases

$2(4.8 \%)$

Digestive system diseases

$1(2.4 \%)$

Arteries/pulmonary circulation diseases $\quad 1(2.4 \%)$

Unknown/ill-defined causes

$14(33.3 \%)$

${ }^{a}$ Cause of death according to death certificate or medical records. 


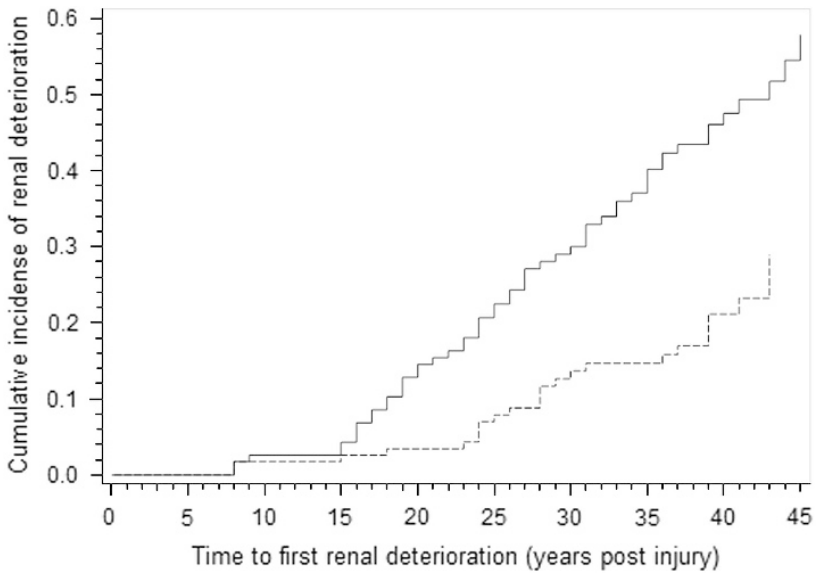

Figure 2 Cumulative incidence curves for moderate (solid line) and severe renal deterioration (dotted line).

the SCI, one patient owing to kidney cancer (hypernephroma), and three owing to multiple ureter/renal stones.

\section{Cumulative incidence of renal deterioration}

The mean ( \pm s.d.) number of renographies and GFR measurements carried out for each patient was $9( \pm 4)$ and $2( \pm 2)$, respectively.

Figure 2 shows the cumulative incidence curves for onset of moderate and severe renal deterioration. Analyses were terminated at 45 YPI (years postinjury), after which the number of patients was $<10$. The cumulative incidence of moderate renal deterioration increases with a constant rate after 15 YPI and is $58 \%$ after 45 YPI. Severe renal deterioration occurs primarily after 23 YPI, and the cumulative incidence is $29 \%$ after 45 YPI.

Table 2 shows the probability of renal deterioration after 20, 30 and 40 YPI.

\section{Risk indicators}

Table 3 shows the univariate effect of different covariates on moderate/ severe renal deterioration and on r-GFR. Dilatation of UUT and a history of renal/ureter stones requiring removal were significant risk indicators of renal deterioration in all analyses. Patients who had sustained an SCI in the period 1970 - 1975 had a significantly higher risk of moderate renal deterioration than patients who had sustained an SCI in 1956-1969, but no difference was found between the groups in terms of severe renal deterioration or the level of r-GFR. Age at injury, gender, level of injury, completeness by the Frankel grade, the predominant bladder-emptying method and the occurrence of bladder stones did not reach statistical significance.

Table 4 shows the results of multivariate analyses examining risk indicators associated with renal deterioration, using Cox proportional hazards for all 116 patients and linear regression for the 83 patients with a GFR measurement. Only the most significant covariates from the univariate analyses in Table 3 were included: level, dilatation of UUT, and renal/ureter stones with and without removal. The proportional hazard analyses were adjusted to the year of SCI. A history of renal/ureter stone requiring removal significantly increased the risk of moderate and severe renal deterioration, hazard ratio (HR) 2.64 and 6.39, respectively, and significantly decreased the level of r-GFR with an unstandardised coefficient of -18.3 $(P=0.003)$. A total of 22 patients had a renal/ureter stone and renal deterioration. The stone was diagnosed before onset of renal deterioration in six patients $(27 \%)$ and after onset of renal
Table 2 Probability of renal deterioration 20, 30 and 40 years after spinal cord injury on cumulative incidence curves

\begin{tabular}{lcc} 
Years postinjury & $\begin{array}{c}\text { Probability of moderate renal } \\
\text { deterioration (\%) }\end{array}$ & $\begin{array}{c}\text { Probability of severe renal } \\
\text { deterioration (\%) }\end{array}$ \\
\hline 20 & 14.5 & 3.4 \\
30 & 30.0 & 13.6 \\
40 & 47.5 & 21.1 \\
\hline
\end{tabular}

deterioration in six patients $(27 \%)$. In three patients (14\%), the renal/ureter stone and renal deterioration occurred within the same year. Renal examinations had not been carried out before the renal/ ureter stone was diagnosed in seven patients (32\%); hence, the order of renal deterioration and renal/ureter stone could not be determined in this group.

Dilatation of UUT significantly increased the risk of moderate renal deterioration with HR 2.20 and severe renal deterioration with HR 5.68. A decreased level of r-GFR (unstandardised coefficient of -8.2) was observed in patients with dilatation of the UUT, although this was not statistically significant $(P=0.07)$. Patients with thoracic-lumbar SCI had a fourfold higher risk of severe renal deterioration compared with patients with cervical SCI $(P=0.005)$, but no association was found in the analysis on moderate renal deterioration or r-GFR.

Figures $3 \mathrm{a}$ and $\mathrm{b}$ show the cumulative incidence of moderate and severe renal deterioration when stratified by dilatation of UUT alone and in combination with a history of renal/ureter stone. Compared with patients with dilatation of the UUT, patients with dilatation of the UUT in combination with a history of renal/ureter stone have a 1.6-fold higher risk of moderate renal deterioration (HR 1.62; 95\% CI, $0.85-3.10 ; P=0.14)$ and a 4.2 -fold higher risk of severe renal deterioration (HR 4.20; 95\% CI, $1.65-10.66 ; P=0.002$ ), after adjusting for level and year of SCI.

\section{DISCUSSION}

In this study, we found an increasing cumulative incidence of renal deterioration over a follow-up period of up to 45 years. The cumulative risk at 45 YPI was $58 \%$ for moderate renal deterioration and $29 \%$ for severe renal deterioration. To our knowledge, the cumulative risk of renal deterioration has not been investigated in an SCI population; hence, a direct comparison of results is not possible. However, Greenwell et al. ${ }^{2}$ found that $21 \%$ of 219 SCI patients had a creatinine clearance $\leqslant 60 \mathrm{ml} \mathrm{min}^{-1}$ with an average injury duration of 21 years and a mean age of $54 \pm 14$ years. In order to compare results, the prevalence of renal deterioration at the twenty-first year after injury for all patients in our study was calculated: $15.5 \%$ had moderate renal deterioration, and 3.4\% had severe renal deterioration with a mean age of $43 \pm 8$ years at 21 YPI. In comparison, $2 \%$ in a $40-59$-year-old cohort in the general population had an estimated GFR $<60 \mathrm{ml} \mathrm{min}{ }^{-1} 1.73 \mathrm{~m}^{-2}, 12$ indicating an increased risk of renal deterioration in an SCI population.

It is well established that GFR decreases with age in able-bodied persons, and not surprisingly, several studies have found a significant effect of age on the level of creatinine clearance and effective renal plasma flow (ERPF) in SCI patients. ${ }^{3,13-15}$ We found no significant association between age at injury and renal deterioration in the univariate analysis, which is explained by the age- and gender-adjusted outcome, r-GFR, we used in our analysis. As we adjusted for the direct effect of age on GFR, the long-term effect of SCI is demonstrated, revealing an increased risk of renal 
Table 3 Univariate analysis of risk indicators associated with renal deterioration

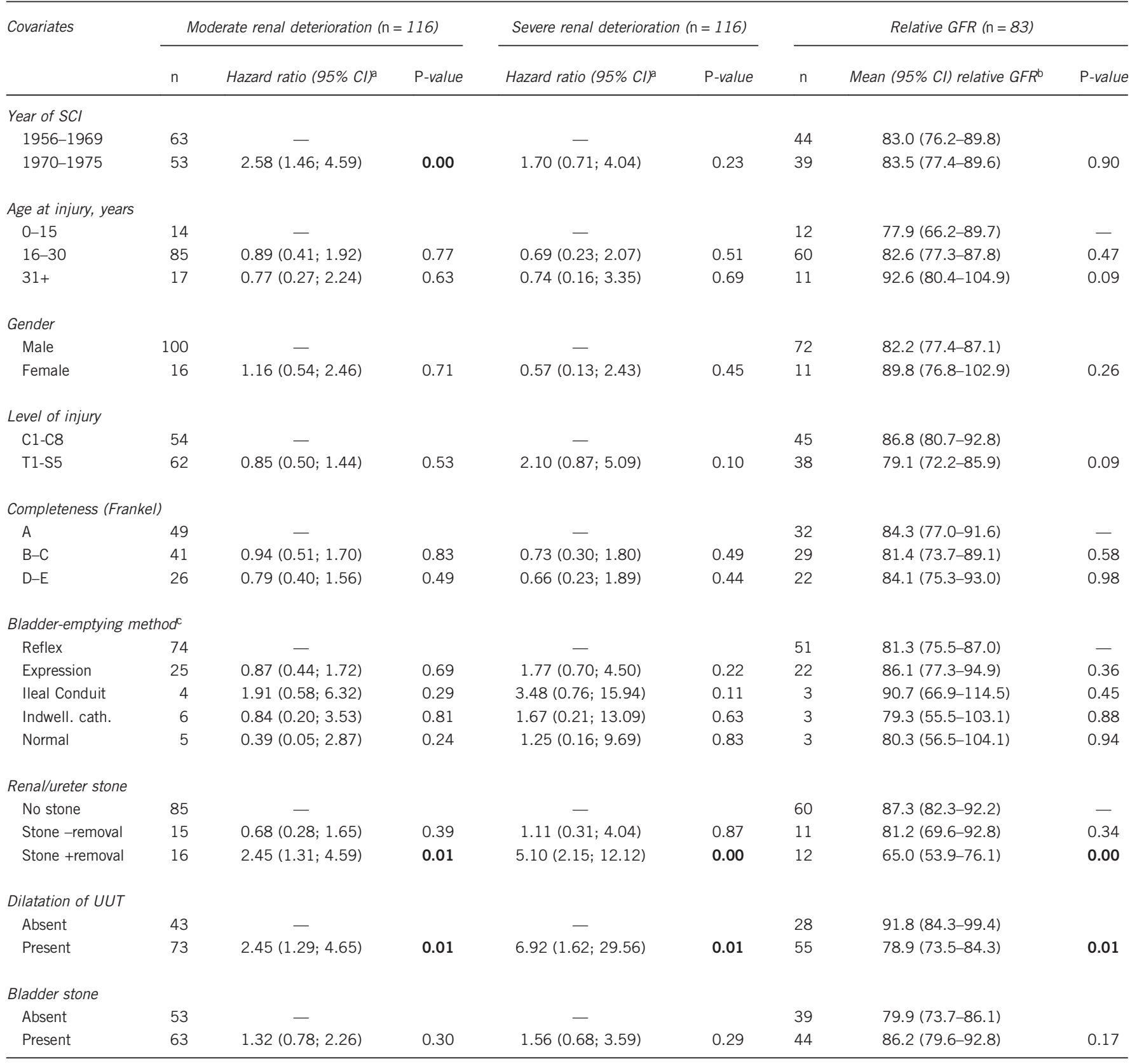

Abbreviations: GFR, glomerular filtration rate; SCI, spinal cord injury; UUT, upper urinary tract. Statistical significant values are indicated in bold.

Statistical significant values are indic
${ }^{a}$ Cox proportional hazards model.

bUnpaired $t$-test, when two catagories, and one-way analysis of variance when $>2$ catagories.

${ }^{c}$ Analyzed without the two patients using clean intermittent catheterization.

deterioration with increasing time after SCI, especially after the twentieth YPI. However, it is a limitation that information on hypertension and diabetes mellitus diagnosed after the time of SCI was not included in this study, as the frequency of these conditions increases with ageing. ${ }^{12}$

Previous studies have assessed long-term renal deterioration in SCI patients using creatinine clearance, ${ }^{2,15}$ ERPF measured by renography ${ }^{13,14,16}$ or a split renal function on renography $\leqslant 40 \%$ in one kidney. ${ }^{15,17}$ A great advantage of this study is the use of ${ }^{51} \mathrm{Cr}$-EDTA clearance. Compared with the gold standard renal inulin clearance, plasma ${ }^{51} \mathrm{Cr}$-EDTA clearance is found to be an accurate method in determining GFR. In addition, ${ }^{51} \mathrm{Cr}$-EDTA clearance does not require urine collection, which makes it more applicable in daily clinical practice. Adversely, the method involves ionising radiation, but the radiation dose is negligible, approximately $0.008 \mathrm{mSv}$ compared with $0.02 \mathrm{mSv}$ from a plain chest radiograph. ${ }^{18,19}$

There was no significant effect of the covariates gender, level of injury and Frankel grade on renal deterioration in this study. Likewise, Weld et al. ${ }^{15}$ found no effect of level and completeness of injury on the level of serum creatinine and creatinine clearance in a study with a 
Table 4 Multivariate analysis of risk indicators associated with different definitions of renal deterioration

\begin{tabular}{|c|c|c|c|c|c|c|}
\hline \multirow[t]{2}{*}{ Risk indicators } & \multicolumn{2}{|c|}{ Moderate renal deterioration $(n=116)$} & \multicolumn{2}{|c|}{ Severe renal deterioration $(n=116)$} & \multicolumn{2}{|c|}{ Relative GFR $(n=83)$} \\
\hline & Hazard ratio $(95 \% \mathrm{Cl})$ & P-value & Hazard ratio $^{\mathrm{a}}(95 \% \mathrm{Cl})$ & P-value & Coefficient $^{b}$ (B) & P-value \\
\hline $\mathrm{C} 1-\mathrm{C} 8$ & - & & - & & - & \\
\hline T1-S5 & $1.07(0.62 ; 1.85)$ & 0.81 & $4.04(1.51 ; 10.8)$ & 0.005 & 6.2 & 0.13 \\
\hline No stone & - & & - & & - & \\
\hline Stone without removal & $0.80(0.32 ; 1.97)$ & 0.62 & $1.65(0.44 ; 6.23)$ & 0.46 & -3.5 & 0.56 \\
\hline Stone with removal & $2.64(1.31 ; 5.33)$ & 0.007 & $6.39(2.23 ; 18.3)$ & $<0.001$ & -18.3 & 0.003 \\
\hline \multicolumn{7}{|l|}{ Dilatation of UUT } \\
\hline Absent & - & & - & & - & \\
\hline
\end{tabular}

Abbreviations: GFR, glomerular filtration rate; SCl, spinal cord injury; UUT, upper urinary tract.

Statistical significant values are indicated in bold.

a Multivariate hazards ratio analyses, adjusted to the year of $\mathrm{SCl}$.

Linear regression analysis describing the unstandardised coefficient $(B)$.
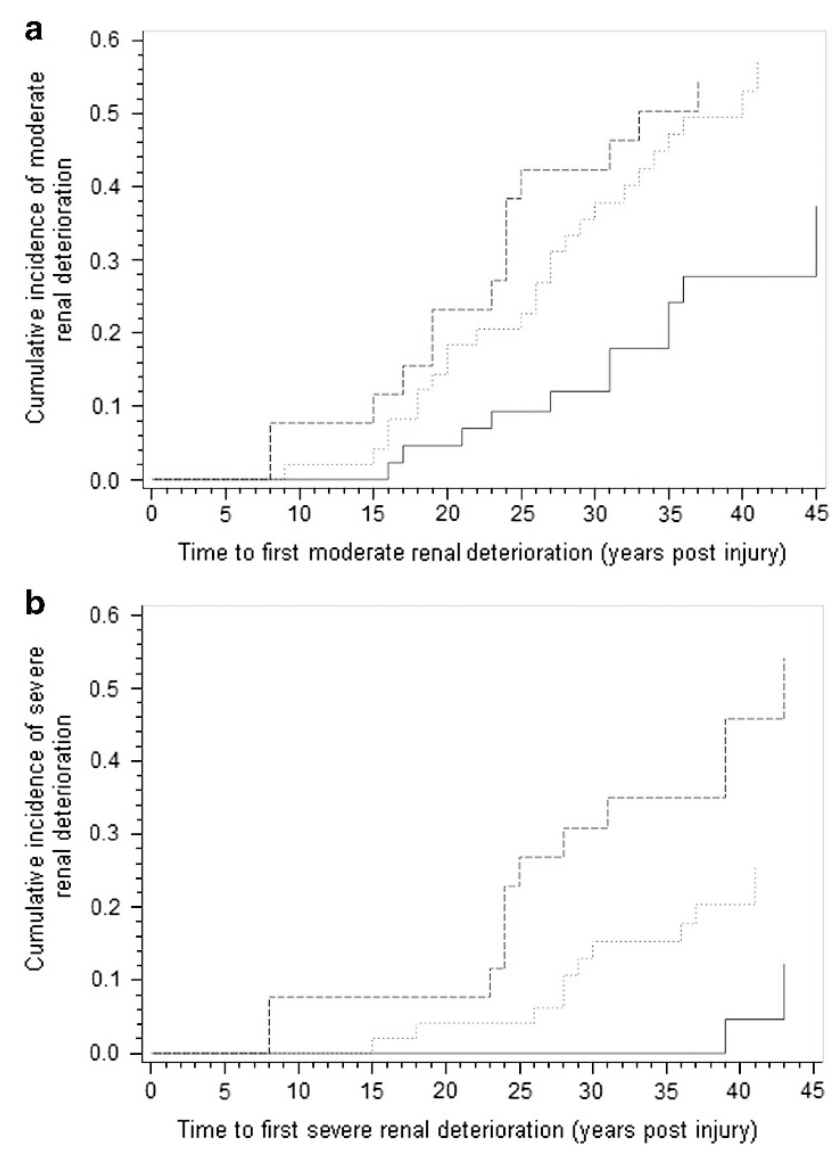

Figure 3 Cumulative incidence curve of renal deterioration stratified by dilatation of UUT with and without a history of renal/ureter stone for (a) moderate renal deterioration and (b) severe renal deterioration. Patients with no dilatation of UUT; ...... patients with dilatation of UUT; _ _ _ patients with dilatation of UUT and a history of renal/ureter stone. mean follow-up of 18.7 YPI. In 1985, Kuhlemeier et al. ${ }^{16}$ reported that high level of SCI and male gender significantly decreased ERPF after a follow-up on maximum 10 YPI. In a study by Sekar et al. ${ }^{13}$ with a follow-up of maximum 21 YPI, completeness and level of the injury had no effect on renal deterioration measured with ERPF, but women had a significantly lower ERPF. Unlike the study by Sekar et al., ${ }^{13}$ we use a gender-adjusted r-GFR as outcome and found no difference between the genders. It should be noted that female patients comprise only $13.8 \%$ of the study population, making the group too small to obtain statistically meaningful results. It could be hypothesised that male SCI patients are at greater risk of renal deterioration compared with females, as the internal sphincter and prostatic urethra more frequently lead to obstructed bladder outlet, especially when using Credé or reflex triggering as the bladder-emptying method. This would result in a higher voiding pressure, which is known to increase the risk of renal deterioration..$^{20,21}$

In the present study, dilatation of UUT included hydronephrosis diagnosed with intravenous pyelographies and delayed excretion on renographic time-activity curves. Dilatation of UUT can appear as a consequence of reflux, although other pathological conditions can cause dilatation of UUT also, for example, ureter stones. Several studies have reported that vesico-uretheral reflux is one of the most important risk indicators of renal deterioration and mortality in SCI patients. ${ }^{4,6}$

We found that a history of renal/ureter stones requiring removal was significantly associated with a decreased level of r-GFR, compared with patients with no history of renal stones. Similar results have been reported. ${ }^{13,16}$ Kuhlemeier et al. ${ }^{16}$ found a mean decrease in ERPF of $51.0 \mathrm{ml} \mathrm{min}{ }^{-1}$ in SCI patients with a history of renal stones. The fact that a history of renal/ureter stone was only a significant risk indicator of renal deterioration, when accompanied by removal of the stone, can be explained by the difference in size of the stone or the fact that removal of a stone is more likely to be carried out if the stone is accompanied by renal deterioration. In our study, renal/ureter stones occur both prior to and after the onset of renal deterioration, suggesting that renal/ureter stones could appear as a consequence of renal deterioration, as well as a risk factor for renal deterioration; 
further studies on a larger cohort are needed in order to make general conclusions.

Indwelling urethral or suprapubic catheter has been reported as the bladder-emptying method with the highest risk of UUT deterioration, as it increases the risk of hydronephrosis, dilatation of UUT, split renal function $\leqslant 40 \%$ in one kidney, decreased creatinine clearance and the presence of proteinuria. ${ }^{6,11,15,20}$ In contrast to these finding, Sekar et al. ${ }^{13}$ did not find any association between the bladder-emptying method at discharge and a decreased level of ERPF in 1114 SCI patients with a maximum follow-up of 21 YPI. We were not able to find any statistically significant association between the bladderemptying method and renal deterioration. As the patients in our study were assigned a predominant bladder-emptying method used for the majority of time until renal deterioration, $85 \%$ of the patients in this study used methods no longer recommended: bladder reflex triggering $(63 \%)$ and bladder straining such as the Valsalvas or Credé manoeuvres (22\%). Only six patients used indwelling catheter and two patients clean intermittent catheterisation (CIC), making the groups too small to obtain statistically meaningful results. Although CIC is recommended for most SCI patients today, studies have shown that a relatively large group of SCI patients change their bladder-emptying method after discharge to reflex voiding or bladder straining. ${ }^{22}$ As the majority of patients in our study used these bladder-emptying methods, the conclusions of this study are relevant in particular to this group of SCI patients.

The risk of missing data is a limitation in this study. Some patients did not attend routine examinations for years after the injury but were included in the follow-up programme later on. This could lead to an underestimation of renal deterioration in SCI patients. Furthermore, follow-up examinations with renography started in 1980. Before this, plasma creatinine was used routinely, but plasma creatinine is considered a poor detector of early renal deterioration ${ }^{7}$ and was not considered in our analysis. As patients included in our study had sustained an SCI between 1956 and 1975, renal deterioration occurring in the first $0-15$ YPI would be detected with a delay of 5-24 years. This can explain the lack of patients with renal deterioration during the first 15 years after SCI and the increase in onset of moderate renal deterioration at 15 YPI. From other studies, we have seen that renal deterioration also occurs within the first 10 YPI. ${ }^{13,16,17}$

The lack of information on urinary tract infections and data from urodynamic examinations is also a limitation for this study. Valid information on urinary tract infections could not be obtained from medical records, and results from urodynamic examinations were not available for the majority of patients. In a prospective study, urodynamic parameters such as maximum detrusor pressures during the filling and/or voiding phase should be included, as an intravesical leak point pressure $>40 \mathrm{~cm} \mathrm{H}_{2} \mathrm{O}$ has been shown to increase the risk of renal deterioration. ${ }^{20}$

Checking of renal function in SCI patients varies between SCI units and is influenced by different national guidelines and differences in health-care systems and patient insurance policies. We acknowledge that the optimal programme is not well defined. An ambitious followup programme has been proposed by the European Association of Urology, recommending lifelong follow-up examinations of the renal function and the urinary tract system every $1-2$ years. ${ }^{4}$ On the basis of our study and the experience from our follow-up programme using renography every second year as a screening examination and ${ }^{51} \mathrm{Cr}$-EDTA clearance as an additional investigation, we find this approach to be sensitive in terms of detecting renal deterioration at an early stage. This gives us the opportunity of changing bladder management to CIC, which is the gold standard for management of neurogenic lower urinary tract dysfunction, ${ }^{4}$ or prescribing medication or other therapeutic interventions in order to preserve the renal function.

In summary, this study supports the need for lifelong evaluation of the renal function in patients with SCI. Renal examinations should be carried out regularly, especially in patients using non-recommended bladder-emptying methods or patients presenting with dilatation of UUT and/or a history of renal/ureter stones requiring removal.

\section{DATA ARCHIVING}

There were no data to deposit.

\section{CONFLICT OF INTEREST}

Marlene Elmelund has received personal fees from Astellas Pharma, outside the submitted work. The other authors declare no conflict of interest.

\section{ACKNOWLEDGEMENTS}

We thank Dr Bonne San Bon Lee from the Department of Spinal Cord Injuries at Prince of Wales Hospital and NEURA, Sydney, Australia for inspiration during the writing process of this paper. The study has been supported by grants from the Lundbeck foundation, grant number 2012-11004. The Lundbeck Foundation had no role in study design, data collection and analysis, decision to publish or preparation of the manuscript.

1 Frankel HL, Coll JR, Charlifue SW, Whiteneck GG, Gardner BP, Jamous MA et al. Long-term survival in spinal cord injury: a fifty year investigation. Spinal Cord 1998; 36: 266-274.

2 Greenwell MW, Mangold TM, Tolley EA, Wall BM. Kidney disease as a predictor of mortality in chronic spinal cord injury. Am J Kidney Dis 2007; 49: 383-393.

3 Abrams P, Agarwal M, Drake M, El-Masri W, Fulford S, Reid S et al. A proposed guideline for the urological management of patients with spinal cord injury. BJU Int 2008; 101: 989-994.

4 Pannek J, Blok B, Castro-Diaz D, Del Popolo G, Kramer G, Radziszewski P et al. Guidelines on Neurogenic Lower Urinary Tract Dysfunction. European Association of Urology 2013 (http://uroweb.org/wp-content/uploads/20_Neurogenic-LUTD_LR.pdf) (accessed 27 October 2015).

5 Cameron AP, Rodriguez GM, Schomer KG. Systematic review of urological followup after spinal cord injury. J Urol 2012; 187: 391-397.

$6 \mathrm{Ku} \mathrm{JH}$, Choi WJ, Lee KY, Jung TY, Lee JK, Park WH et al. Complications of the upper urinary tract in patients with spinal cord injury: a long-term follow-up study. Urol Res 2005; 33: 435-439.

7 Elmelund M, Oturai PS, Biering-Sorensen F. 50 years follow-up on plasma creatinine levels after spinal cord injury. Spinal Cord 2014; 52: 368-372.

8 Brochner-Mortensen J, Jensen S, Rodbro P. Delimitation of plasma creatinine concentration values for assessment of relative renal function in adult patients. Scand J Urol Nephrol 1977; 11: 257-262.

9 Brochner-Mortensen J, Hammerich B, Christoffersen J. Assessment of renal function from plasma urea and plasma creatinine in children. Scand J Urol Nephrol 1982; 16: 229-236.

10 Frankel HL, Hancock DO, Hyslop G, Melzak J, Michaelis LS, Ungar GH et al. The value of postural reduction in the initial management of closed injuries of the spine with paraplegia and tetraplegia. I. Paraplegia 1969; 7: 179-192.

11 Weld KJ, Dmochowski RR. Effect of bladder management on urological complications in spinal cord injured patients. J Urol 2000; 163: 768-772.

12 Coresh J, Astor BC, Greene T, Eknoyan G, Levey AS. Prevalence of chronic kidney disease and decreased kidney function in the adult US population: Third National Health and Nutrition Examination Survey. Am J Kidney Dis 2003; 41: 1-12.

13 Sekar P, Wallace DD, Waites KB, DeVivo MJ, Lloyd LK, Stover SL et al. Comparison of long-term renal function after spinal cord injury using different urinary management methods. Arch Phys Med Rehabil 1997; 78: 992-997.

14 Waites KB, Canupp KC, DeVivo MJ, Lloyd LK, Dubovsky EV. Compliance with annual urologic evaluations and preservation of renal function in persons with spinal cord injury. J Spinal Cord Med 1995; 18: 251-254.

15 Weld KJ, Wall BM, Mangold TA, Steere EL, Dmochowski RR. Influences on renal function in chronic spinal cord injured patients. J Urol 2000; 164: 1490-1493.

16 Kuhlemeier KV, Lloyd LK, Stover SL. Long-term followup of renal function after spinal cord injury. J Urol 1985; 134: 510-513.

17 Biering-Sorensen F, Nielans HM, Dorflinger T, Sorensen B. Urological situation five years after spinal cord injury. Scand J Urol Nephrol 1999; 33: 157-161. 
18 Medeiros FS, Sapienza MT, Prado ES, Agena F, Shimizu MH, Lemos FB et al. Validation of plasma clearance of 51Cr-EDTA in adult renal transplant recipients: comparison with inulin renal clearance. Transpl Int 2009; 22: 323-331.

19 Soveri I, Berg UB, Bjork J, Elinder CG, Grubb A, Mejare I et al. Measuring GFR: a systematic review. Am J Kidney Dis 2014; 64: 411-424.

20 McGuire EJ, Woodside JR, Borden TA, Weiss RM. Prognostic value of urodynamic testing in myelodysplastic patients. J Urol 1981; 126: 205-209.
21 Rule AD, Jacobson DJ, Roberts RO, Girman CJ, McGree ME, Lieber MM et al. The association between benign prostatic hyperplasia and chronic kidney disease in community-dwelling men. Kidney Int 2005; 67 2376-2382.

22 Afsar SI, Yemisci OU, Cosar SN, Cetin N. Compliance with clean intermittent catheterization in spinal cord injury patients: a long-term follow-up study. Spinal Cord 2013; 51: 645-649. 